\title{
A Comment on Reis*
}

\author{
Kenji Miyazaki \\ Faculty of Economics, Hosei University, Tokyo, Japan \\ E-mail:miya_ken@hosei.ac.jp \\ Received July 17, 2011; revised August 31, 2011; accepted September 8, 2011
}

\begin{abstract}
This note gives a counterexample on Reis [1]. Using a certain family of utility functions, this note not only gives a sharper representation than that of Reis but also demonstrates that interest rate inelastic money demand does not lead to superneutrality. This implies that superneutrality does not exist when uncerinty is introduced.
\end{abstract}

Keywords: Monetary Policy, Superneutrality, Nominal Interest Rate Policy, Perfect Complementary between Consumption and Money

\section{Introduction}

Reis [1] characterized the dynamics of the money-in-thetility model (Sidrauski, [2]) by using the money demand function to explain the mechanism in a very intuitive manner. One of his main conclusions is that when asming that the government can control nominal interest rates by setting any growth rate of money supply, monetry policy does not affect any level of consumption and capital stock as long as either money demand is inelastic with respect to nominal interest or money and consumption are separable in the utility function. Subsequently, Lioui and Poncet [3] attached uncertainty with Reis' framework to demonstrate that superneutrality is valid only in the case of an interest rate inelastic money demand. However, both studies do not pursue a sufficient investigation on the relationship between the money demand function and the utility function.

This note gives a counterexample for their statements. That is, we show that within a certain family of utility functions, interest rate inelastic money demand does not lead to superneutrality. An intuitive explanation is as follows. A nominal interest monetary policy affects real variables through the product of the interest rate elasticity of money demand and the elasticity of the marginal utility of consumption with respect to money. When conmption and money are perfectly complementary, the former elasticity is zero but the latter elasticity takes infinity. When

\footnotetext{
*The author is grateful for a grant-in-aid from the Ministry of Education and Science, the Government of Japan (21530277).

${ }^{1}$ In the conventional monetary policy with a constant rate of money growth $\mu$, we should add $\eta \dot{R} / R+\zeta \dot{c} / c=f_{k}+\mu-R$ to the two equations in order to describe the system.
}

the product of both elasticities converges to a finite value, such a policy is still effective.

\section{S-Sidrauski Economy}

In order to prepare a counterexample, this section briefly reviews a Reis-Sidrauski economy and reconsiders the assumptions on the utility function of Reis [1].

In the economy, $c_{t}>0, k_{t}>0$, and $m_{t}>0$, respecttively, denote consumption, capital stock, and real balances or just money. Technology is characterized by a constant parameter $\delta>0$ of depreciation rate and a production function $f\left(k_{t}\right)<0$ with $f_{k}>0, f_{k k}<0$, $f(0)=0, \lim _{k \rightarrow 0} f_{k}=\infty$, and $\lim _{k \rightarrow \infty} f_{k}=0$. Representative agents are infinity lived with perfect foresight, and their preferences are characterized by a constant parameter $\rho>0$ of the rate of time preference and a utility function $u\left(c_{t}, m_{t}\right)$.

A set of assumptions imposed on $u$ is discussed later. In equilibrium, the representative agent maximizes their lifetime utility to choose $c_{t}$ and $m_{t}$, the markets are clear, and the government chooses nominal interest rates $R_{t}=f_{k}\left(k_{t}\right)+\pi_{t}$, where $\pi_{t}$ denotes inflation rates, by controlling an appropriate rate of money growth.

The equilibrium dynamics system is characterized by the money demand function $\varphi(c, R)$, defined by $R=u_{m}(c, \varphi) / u_{c}(c, \varphi)$, which results from the necessary condition for the maximization problem of the representative agents. Using $\varphi$, we describe the dynamics system ${ }^{1}$ as

$$
\begin{aligned}
\theta \frac{\dot{c}}{c} & =f_{k}-\delta-\rho-\xi \eta \frac{\dot{R}}{R} \\
\dot{k} & =f-\delta k-c .
\end{aligned}
$$


where

$$
\begin{aligned}
& \theta=-c u_{c c}(c, \varphi(c, R)) / u_{c}(c, \varphi(c, R))-\xi \zeta, \\
& \xi=m u_{c m}(c, \varphi(c, R)) / u_{c}(c, \varphi(c, R)), \\
& \eta=-R \varphi_{R}(c, R) / \varphi(c, R), \\
& \zeta=c \varphi_{c}(c, R) / \varphi(c, R),
\end{aligned}
$$

respectively, represent the inverse of the intertemporal elasticity of substitution, the elasticity of the marginal utility of consumption with respect to real money balances, the interest rate elasticity of money demand, and the consumption elasticity of money demand.

Reis [1], in his proposition 2, stated that money is superneutral when $\xi \eta$ is equal to zero. Following the proposition, Reis stated that such superneutrality attains either if money and consumption are separable in the utility function $(\xi=0)$ or if money demand is inelastic with respect to nominal interest $(\eta=0)$. In this note, we give a counterexample satisfying $\eta=0$ but $\xi \eta \neq 0$.

Before providing the example, we discuss a set of assumptions regarding the utility function. Reis [1] assumed $u_{c}>0, u_{c c}<0, u_{m} \geq 0, u_{m m} \leq 0, u_{c c} u_{n n} \geq u_{c m}$, and $u_{c m} \geq 0$. When we assume $u_{m}=0$, then

$u_{m} / u_{c}=R=0$, implying that the government should set zero nominal interest rates. In addition, when we assume $u_{c c} u_{m m}=u_{c m}^{2}$, then, as shown later, we cannot exclude the possibility of $\theta=0$. The assumption $u_{c m} \geq 0$ is a little bit restrictive because this assumption excludes the case of $\theta>1$ in the famous CRRA form of $u(c, m)=\left(c^{1-\alpha} m^{\alpha}\right)^{1-\theta} /(1-\theta)$, where $0<\alpha<1$ is a constant parameter.

Instead of the above assumptions on the utility function, we propose the following assumption: $u_{c}>0$, $u_{c c}<0, u_{m}>0, u_{m m}<0, u_{c c} u_{n n}-u_{c m}^{2}>0$, $u_{c m} u_{m}-u_{m m} u_{c}>0$, and $u_{c} u_{c m}-u_{c c} u_{m}>0$ for all $c>0$ and $m>0$. The first four assumptions indicate that $u$ is strictly increasing and strictly concave with respect to $c$ and $m$. The last two assumptions arise from $\partial\left(u_{m} / u_{c}\right) / \partial c>0$ and $\partial\left(u_{m} / u_{c}\right) / \partial m>0$. These assumptions are the same as those of Fischer [4]. Using the total differential form:

$$
\mathrm{d} R=\left\{\partial\left(u_{m} / u_{c}\right) / \partial c\right\} d c+\left\{\partial\left(u_{m} / u_{c}\right) / \partial m\right\} \mathrm{d} m,
$$

we obtain

$$
\begin{gathered}
\varphi_{R}=\left.\frac{u_{c}^{2}}{u_{m m} u_{c}-u_{c m} u_{m}}\right|_{m=\varphi(c, R)} \\
\varphi_{c}=\left.\frac{u_{c c} u_{m}-u_{c} u_{c m}}{u_{m m} u_{c}-u_{c m} u_{m}}\right|_{m=\varphi(c, R)}
\end{gathered}
$$

\footnotetext{
${ }^{2}$ In fact, $\frac{\mathrm{d}}{\mathrm{d} z} \frac{\psi(z)}{\psi(z)-z \psi^{\prime}(\mathrm{z})}=\frac{\psi^{\prime \prime}(z) \psi(z)}{\left\{\psi(z)-z \psi^{\prime}(z)\right\}^{2}}<0$

${ }^{3}$ See Appendix.
}

and

$$
\theta=-\left.c \frac{u_{c c} u_{m m}-u_{c m}^{2}}{u_{m m} u_{c}-u_{c m} u_{m}}\right|_{m=\varphi(c, R)} .
$$

Therefore, if the above assumptions are satisfied, then $-\varphi_{R}, \varphi_{c}$, and $\theta$ are all nonnegative. When $u_{c m} u_{m}-u_{m m} u_{c}$ and $u_{c} u_{c m}-u_{c c} u_{m}$ are finite, then $-\varphi_{R}, \varphi_{c}$, and $\theta$ are all positive.

From equation (1), the interest rate elasticity of money demand $\eta=-R \varphi_{R} / \varphi$ might takes zero only when $u_{c m} u_{m}-u_{m m} u_{c}$ takes infinity, This would happen when $u_{c m}$ or $\zeta=m u_{c m} / u_{c}$ takes infinity. This makes us conjecture that, even when $\eta=0$, the product of $\eta$ and $\zeta$ is not necessarily zero.

\section{Counter Example}

Because we cannot prove the conjecture in the above general class of utility functions, we set a somewhat restrictive class to give a counterexample. Let

$u(c, m)=w(c \psi(z))$, where $z=m / c>0$. When

$-c \psi w^{\prime \prime} / w^{\prime}$ is constant, this is exactly the class of utility functions Lucas [5] proposed. In order for $u$ to be strictly increasing and strictly concave with respect to $c$ and $m$, respectively, we assume that $w$ and $\psi$ are strictly increasing and strictly concave, respectively, and $0<z \psi^{\prime} / \psi<1$ for all $z>0$.

Under this class, the money demand function is determined by

$$
R=\frac{\psi(z)}{\psi(z)-z \psi^{\prime}(z)} .
$$

The right-hand side of the above equation is positive and strictly decreasing for all $z>0,{ }^{2}$ and, accordingly, there exists an inverse function $z=\phi(R)$. Thus, the money demand function $m=c \phi(R)$ is well-defined. The elasticities of the money demand function with respect to consumption and interest rates are, respectively, unity and

$$
\eta=-\frac{R \phi^{\prime}(R)}{\phi(R)}=-\left.\frac{\psi^{\prime}(z)\left\{\psi(z)-z \psi^{\prime}(z)\right\}}{z \psi(z) \psi^{\prime \prime}(z)}\right|_{z=\phi(R)} .
$$

The last equality is established by using Equation (1) and $u(c, m)=w(c \psi(z))$.

The dynamic is described as the same in the previous section and the coefficients are expressed in a simpler way. With some algebraic operations ${ }^{3}$, we can get

$$
\theta=c \psi w^{\prime \prime} /\left.w^{\prime}\right|_{z=\phi(R)}
$$

and

$$
\xi=\left.(1 / \eta-\theta) \frac{z \psi^{\prime}(z)}{\psi(z)}\right|_{z=\phi(R)}
$$


Equation (3) indicates that the elasticity of the shadow price $u_{c}$ with respect to money is represented much more clearly than that of Reis [1]. That is, the elasticity $\zeta$ is determined by $\eta, \theta$, and the relative slope of $\psi$. When $1 / \eta>\theta$ or $\eta<1 / \theta$, then the interest elasticity of money demand is smaller than the elasticity of the intertemporal substitution. In this case, the shadow price of capital is increasing in money. When $\eta=1 / \theta$, then $u_{c m}=0$ or the utility function is separable.

Because $\xi \eta=(1-\eta \theta) z \psi^{\prime} / \psi$ and $0<z \psi^{\prime} / \psi<1$, we can show $\eta=0$ but $\xi \eta \neq 0$ within our family of utility function. Even if $\eta \rightarrow 0, \xi$ is growing much faster, and, accordingly, $\xi \eta$ converges to $z \psi^{\prime} / \psi$. Only when the utility function is separable does $\xi \eta$ take the value of zero.

Finally, we present a parametric example. The utility function is described as

$$
u(c, m)=\frac{\left[(1-\alpha) c^{\frac{\eta-1}{\eta}}+\alpha m^{\frac{\eta-1}{\eta}}\right]^{\frac{\eta(1-\theta)}{\eta-1}}}{1-\theta}=\frac{\{c \psi(\mathrm{z})\}^{1-\theta}}{1-\theta}
$$

where $0<\alpha<1, \theta>0$, and $\eta \geq 0$ are constant parameters and $\psi(z)=\left[1-\alpha+\alpha z^{\frac{\eta-1}{\eta}}\right]^{\frac{\eta}{\eta-1}}$ for $z=m / c>0$. Notice that $z=\min [c, m]$ when $\eta=0$ and that $z=c^{1-\alpha} m^{\alpha}$ when $\eta=1$. Consumption and real balances are perfect complements when $\eta=0$. The case of $\eta=0$ corresponds the case of a cash-in-advance economy, in which money is needed for purchasing consumption goods and the cash-in-advance constraint is always binding ${ }^{4}$.

In this case, the elasticity of intertemporal substitution and the interest rate elasticity are respectively determined by the constant parameters $1 / \theta$ and $\eta$, and $\xi \eta$ is represented as a function only of $R$, or

$$
\xi \eta=\left(\frac{\alpha^{\eta} R^{1-\eta}}{(1-\alpha)^{\eta}+\alpha^{\eta} R^{1-\eta}}\right)(1-\theta \eta) .
$$

Clearly, $\xi \eta=R /(1+R)$ when $\eta=0$ and $\xi \eta=\alpha(1-\theta)$ when $\eta=1$. when $\theta \eta=1, \xi \eta$ takes zero.

\section{Concluding Remarks}

In summary, using a larger set of utility functions than that of Lucas [5], we not only give a sharper representation than that of Reis [1] but also give a counterexample. When consumption and real balances are perfectly complement, then the interest rate elasticity of money demand is zero but a nominal interest policy is not superneutral. Only in the case of a separable utility function does superneutrality survive. This discussion assumes that consumers have perfect foresight and no uncertainty exists. When uncertainty is introduced, following Lioui and Poncet [3], separability does not assure superneutrality. Therefore, no superneutrality exists with our family of utility functions.

\section{References}

[1] R. Reis, "The Analytics of Monetary Non-Neutrality in the Sidrauski Model,” Economics Letters, Vol. 94, No. 1, 2007, pp. 129-135. doi:10.1016/j.econlet.2006.08.017

[2] M. Sidrauski, "The Rational Choice and Patterns of Growth in a Monetary Economy," American Economic Review, Vol. 57, No. 2, 1967, pp. 534-544.

[3] A. Lioui and P. Poncet, "Monetary Non-Neutrality in the Sidrauski Model under Uncertainty,” Economics Letters, Vol. 100, No. 1, 2008, pp. 22-26. doi:10.1016/j.econlet.2007.10.023

[4] S. Fischer, "Capital Accumulation on the Transition Path in a Monetary Optimizing Model,” Econometrica, Vol. 47, No. 6, 1979, pp. 1433-1439. doi:10.2307/1914010

[5] R. E. Lucas Jr., "Inflation and Welfare,” Econometrica, Vol. 68, No. 2, 2000, pp. 247-274. doi:10.1111/1468-0262.00109
${ }^{4}$ The constraint $m \leq c$ is binding when the government sets the nominal interest rate to be positive. 


\section{Appendix}

Consider $u(c, m)=w(y)$, where $y=\psi(m / c) c$. The derivatives of $u$ are described as follows:

$$
\begin{aligned}
& u_{c}=\left\{\psi(m / c)-\psi^{\prime}(m / c) m / c\right\} w^{\prime}(y) \\
& u_{m}=\psi^{\prime}(m / c) w^{\prime}(y) \\
& u_{c c}=\left\{\psi(m / c)-\psi^{\prime}(m / c) m / c\right\}^{2} w^{\prime \prime}(y) \\
&+\left(m^{2} / c^{3}\right) \psi^{\prime \prime}(m / c) w^{\prime}(y) \\
& u_{m m}=\left\{\psi^{\prime}(m / c)\right\}^{2} w^{\prime \prime}(y)+\psi^{\prime \prime}(m / c)(1 / c) w^{\prime}(y) \\
& u_{c m}= \psi^{\prime}(m / c)\left\{\psi(m / c)-\psi^{\prime}(m / c) m / c\right\} w^{\prime \prime}(y) \\
&-\left(m / c^{2}\right) \psi^{\prime \prime}(m / c) w^{\prime}(y) .
\end{aligned}
$$

The money demand function is derived from Equation (2). The total differential form is described as $\mathrm{d} R=\left\{\partial\left(u_{m} / u_{c}\right) / \partial c\right\} \mathrm{d} c+\left\{\partial\left(u_{m} / u_{c}\right) / \partial m\right\} \mathrm{d} m$, where

$$
\frac{\partial\left(u_{m} / u_{c}\right)}{\partial c}=\frac{u_{c} u_{c m}-u_{m} u_{c c}}{u_{c}^{2}}=-\frac{\left(m / c^{2}\right) \psi(m / c) \psi^{\prime \prime}(m / c)}{\left\{\psi(m / c)-(m / c) \psi^{\prime}(m / c)\right\}^{2}}
$$

$$
\frac{\partial\left(u_{m} / u_{c}\right)}{\partial m}=\frac{u_{c} u_{m m}-u_{m} u_{c m}}{u_{c}^{2}}=\frac{(1 / c) \psi(m / c) \psi^{\prime \prime}(m / c)}{\left\{\psi(m / c)-(m / c) \psi^{\prime}(m / c)\right\}^{2}} .
$$

Using $\varphi_{c}=-\left\{\partial\left(u_{m} / u_{c}\right) / \partial c\right\} /\left\{\partial\left(u_{m} / u_{c}\right) / \partial m\right\}=m / c$ and $1 /\left\{\partial\left(u_{m} / u_{c}\right) / \partial m\right\}$, we obtain:

$$
\begin{aligned}
& \varphi_{R}=\eta=-R /\left\{m \partial\left(u_{m} / u_{c}\right) / \partial m\right\} \\
&=-\frac{\psi^{\prime}(m / c)\left\{\psi(m / c)-(m / c) \psi^{\prime}(m / c)\right\}}{(m / c) \psi(m / c) \psi^{\prime \prime}(m / c)} \\
& \zeta=-\left\{c \partial\left(u_{m} / u_{c}\right) / \partial c\right\} /\left\{m \partial\left(u_{m} / u_{c}\right) / \partial m\right\}=1 .
\end{aligned}
$$

Because of $\zeta=1$,

$$
\theta=-\frac{c u_{c c}}{u_{c}}-\frac{m u_{c m}}{u_{c}}=-\frac{c \psi(m / c) w^{\prime \prime}(y)}{w^{\prime}(y)} .
$$

Because of

$$
\frac{m u_{c m}}{u_{c}}=-\theta \frac{m / c \psi^{\prime}(m / c)}{\psi(m / c)}-\frac{\left(m^{2} / c^{2}\right) \psi^{\prime \prime}(m / c)}{\psi(m / c)-\psi^{\prime}(m / c) m / c},
$$

we obtain Equation (3). 